\title{
Broadband Active Phase Shifter GaAs MMIC
}

\author{
Ph. Duême - Th. Dequen - R. Funck - B. Caillon - G. Guerbeur \\ THALES AIRBORNE SYSTEMS \\ 2, avenue Gay-Lussac 78851 Elancourt Cedex, France, philippe.dueme@ fr.thalesgroup.com
}

\begin{abstract}
A broadband multifunction MMIC, achieving combined amplification and phase shift, has been developed on 2 (3.2 $\times 4) \mathrm{mm}^{2}$ chips using the UMS PH25 process. The frequency range is as large as characterised by a ratio of Fmax/Fmin $=6$. The 5 bit phase shifter section is based on switched "all-pass" cells. The amplification function is realised through active switches consuming less than a total of $40 \mathrm{~mA}$ under $3 \mathrm{~V}$ and providing an overall gain of about +6 dB.
\end{abstract}

\section{INTRODUCTION}

When dealing with simultaneous constraints such as very broadband operation of several functions, high performances, low consumption and good linearity, it becomes obvious that specific multifunction GaAs MMICs are the unique solution. It is then necessary to carefully translate system needs into a proper MMIC architecture, taking into account all the capabilities of MMIC technology. This paper describes a phase shifter with integrated amplification, working over a frequency band characterised in the ratio : Fmax/Fmin $=6$. The function is split in 2 circuits to form a 5 bit phase shifter using active and passive switches between original phase shifting passive structures based on all-pass filters realized by interwoven spirals. All the active parts use the distributed structure to achieve broadband operation. Very good results were obtained after a single foundry run using a pHEMT UMS process line (PH25). Each MMIC has a surface area of $4 \mathrm{~mm} \times 3.2 \mathrm{~mm}$. The overall consumption is about $40 \mathrm{~mA}$ under $3 \mathrm{~V}$ for an overall gain of about +6 $\mathrm{dB}$.

\section{OBJECTIVES AND DESIGN}

The specific goal of this design was to achieve good performances of the phase shifter function while maintaining good levels for the overall gain, noise figure at the input, power level and linearity at the output and with a very low power consumption. As depicted on the functional diagram shown in figure 1, the phase shifter is built following a classical structure of switched sub-circuits, each sub-circuit being a combination of 2 "all-pass" cells (or "constant-R" networks). One original point is that the broadband switches are alternately active and passive circuits (triangles in Figure 1 stand for active switches), to maintain a fairly constant power level along the structure and save power consumption. Each active switch is placed so that it compensates for a little bit more than the losses of the following passive components, leading to an overall gain with a positive value of about $6 \mathrm{~dB}$.

Another original feature is the use of interwoven spirals (patent pending) to realize a mutual inductance between 2 spiral inductors.

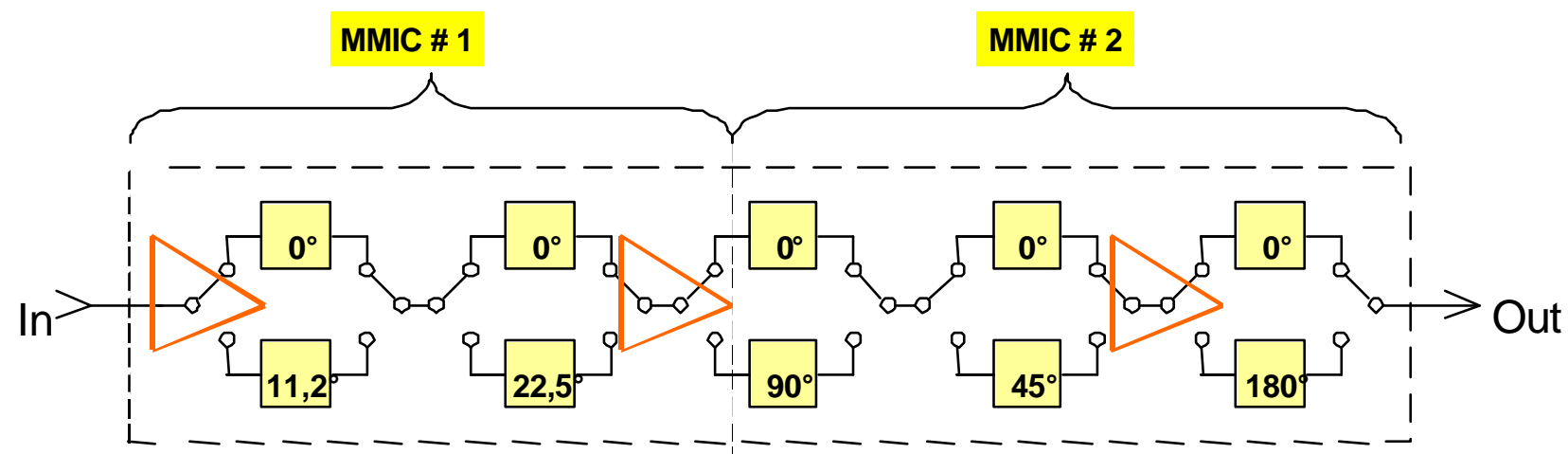




\section{BASIC THEORY OF PHAS E SHIFTING CELLS}

The targeted bandwidth $($ Fmax/Fmin $=6)$ doesn't allow the use of Lange couplers or a structure with switched components. Switched "all-pass" filter cells have been chosen for their ability to cover a broad band. An ideal all-pass cell is presented on Figure 2 associated with some basic equations involving the reference impedance, the transition frequency of the cell (the frequency at which a phase rotation occurs) and the parameters of the cells. Among these parameters, the mutual inductance has a high impact on performances: the use of a high value of mutual inductance can dramatically improve the flatness of the phase difference between 2 all-pass cells, as shown on Figure 4. A value of about 0.6 for the ratio $\mathrm{m}=\mathrm{M} / \mathrm{L}$ can be obtained with interwoven spirals as illustrated on Figure 5. and Figure 6. To further improve the useful frequency band, each bit is constructed of 2 paths containing each 2 all-pass cells (Figure 3), leading to a full phase shifter bit containing 4 cells (the $180^{\circ}$ bit contains 8 cells at this first design step).

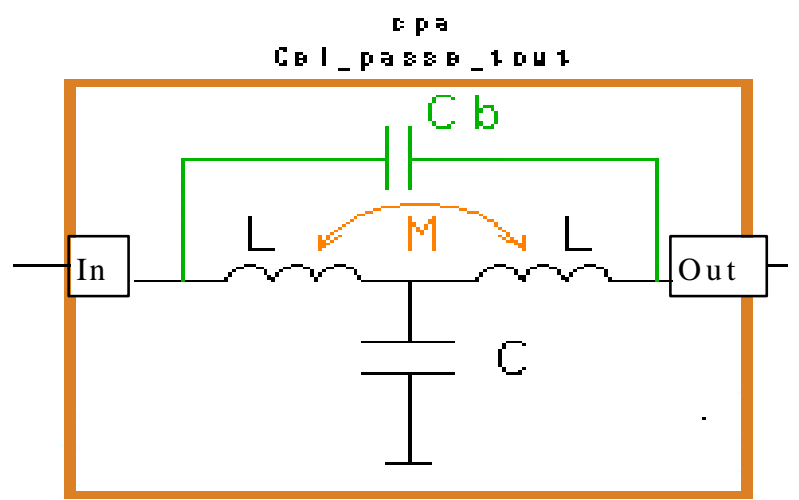

EQUATION $c=2 * k /($ Om0*ZO) EQUATION mrk=(1-k*2)/(1+k*2*2) EQUATION $\mathrm{cb}=\mathrm{c} /(4 * k * * 2)$ EQUATION $k=s q r t((1-m r) /(1+m r))$ EQUATION $m=-m r * 1$

EQUATIOH $I=Z 0 *(1+k * 2) /($ om $0 * 2 * k)$

Figure 2 : one theoretical "all-pass filter" cell
Path A

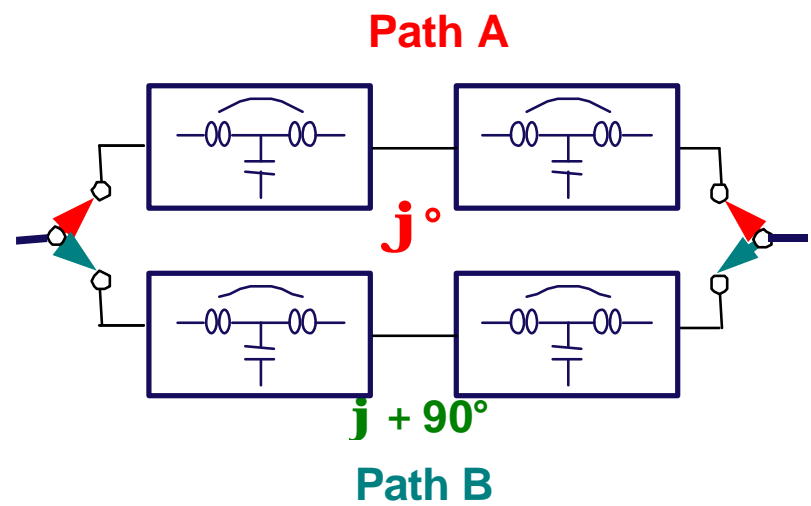

Figure 3 : Schematic diagram of a phase shifting bit made of 2 switched channels containing 2 all-pass cells each

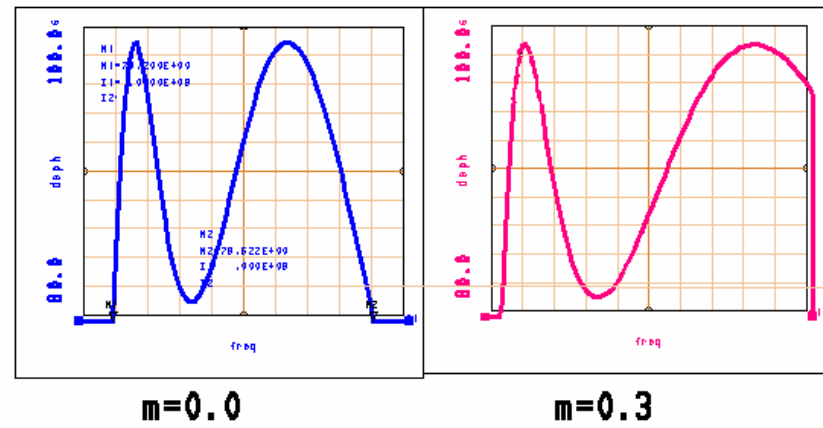

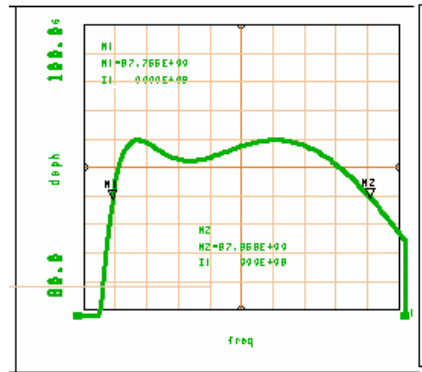

$m=0.5$

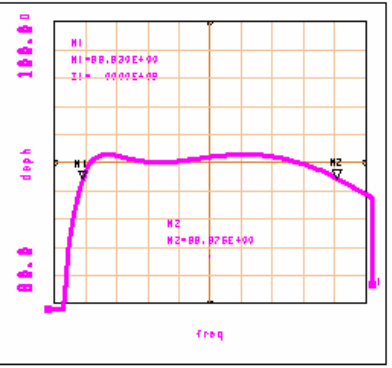

$m=0.6$
Figure 4 : Variation of phase difference ripple versus relative mutual inductance for a $90^{\circ}$ bit following

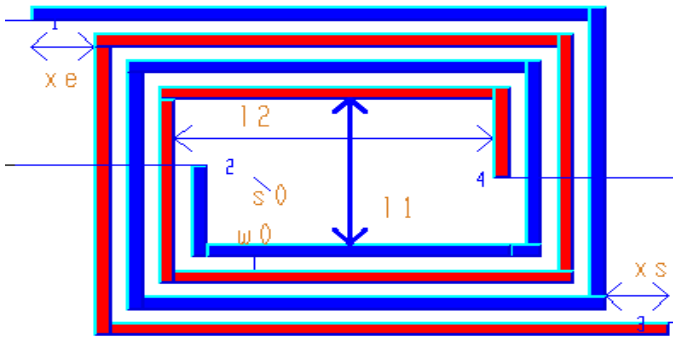

Figure 5 : Symbolic representation of 2 inductances linked by a relatively strong mutual inductance 


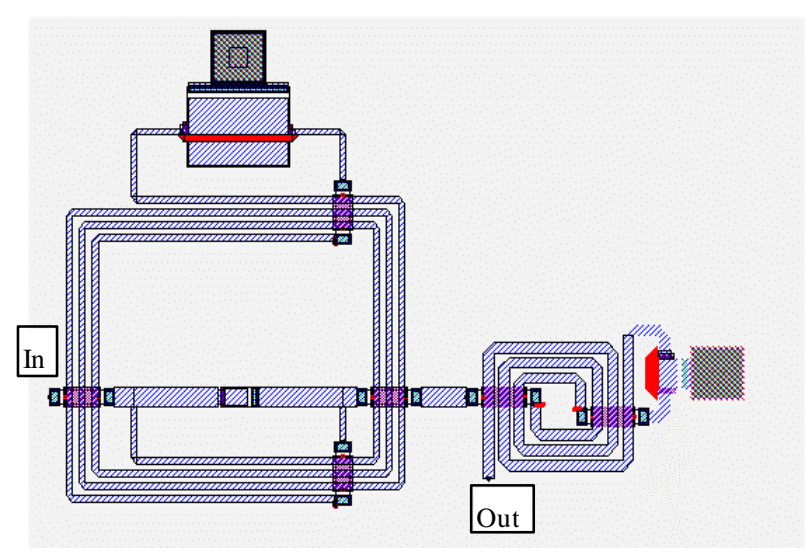

Figure 6: Practical layout of 2 cells to achieve one path of a phase shift bit.

\section{SWITCHES BETWEEN PHASE SHIFTING CELLS}

The cells are switched with either passive (cold FETs) or active circuits. The active parts are based on distributed structures with multiple lines. The first one is a SPDT with one gate line and two drain line. The second one is a fully original structure (patent pending), a DPDT (from any of 2 inputs to any of 2 outputs) with two gate lines and two drain lines, using cascode cells, the common gate FET of which is shared between two switched common source FETs. This circuit is shown on Figure 7.

The active structure, where each FET acts as a switching and an amplifying device at the same time is very efficient to preserve both low noise figure and good dynamic behaviour.

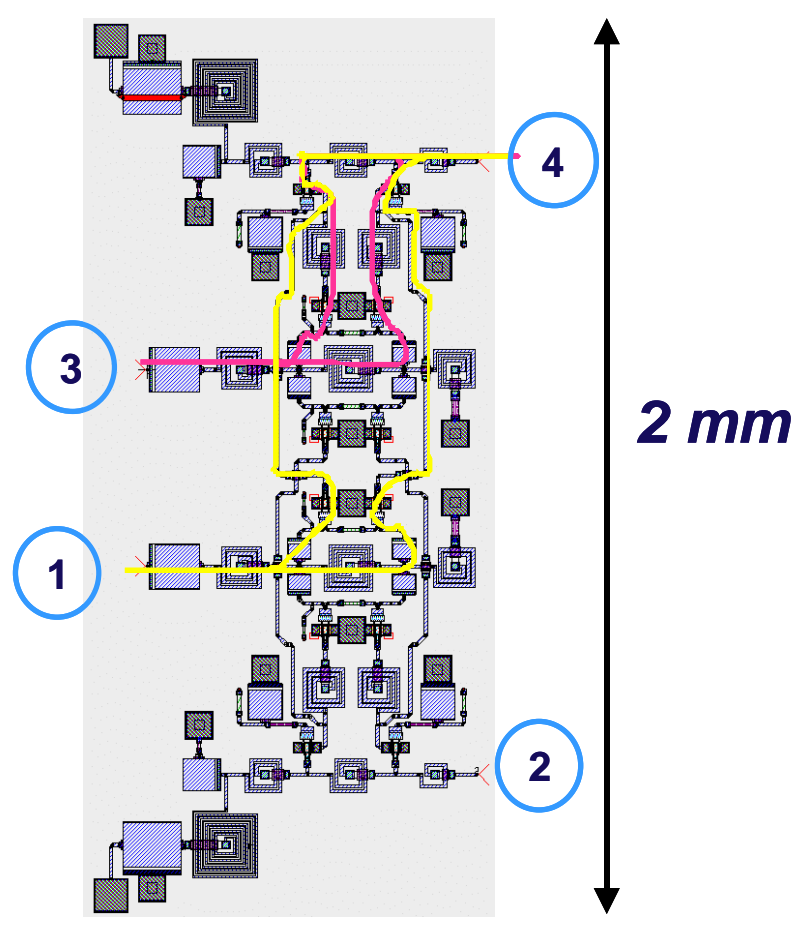

Figure 7 : Layout of an active DPDT with a symbolic representation of the signal flow in 2 different states.

\section{LAYOUT AND RESULTS}

The Figure 8 and Figure 9 show the layout of the circuits (size : $4 \times 3.2 \mathrm{~mm}^{2}$ ). The Figure 10 shows one curve comparing simulation (using an in-house developed software for the interwoven spirals that are completely original devices) and measurement of the phase shift for a $90^{\circ}$ bit. It is highlighted that such a phase shift has been obtained with a single circuit, without the need for splitting the frequency band in sub-bands. The on-chip measurement results for the full circuits are presented on Figure 11 and Figure 12. The gain (around $8 \mathrm{~dB}$ for the first chip and $-2 \mathrm{~dB}$ for the second one) and noise figure (5.5. dB) are also extremely well simulated, thanks to the good quality of the models provided by the UMS foundry. 


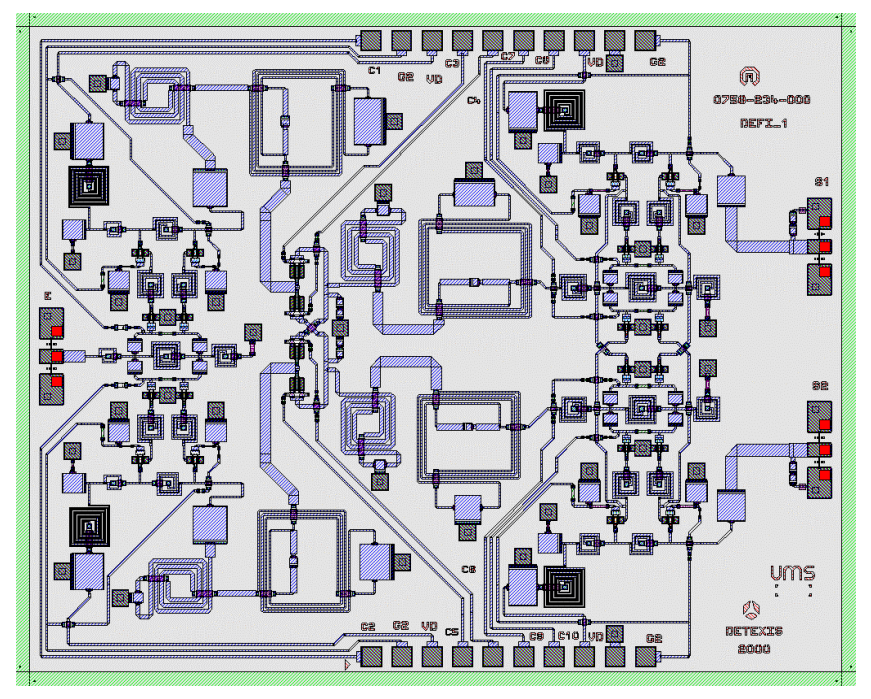

Figure 8: Layout of the first MMIC

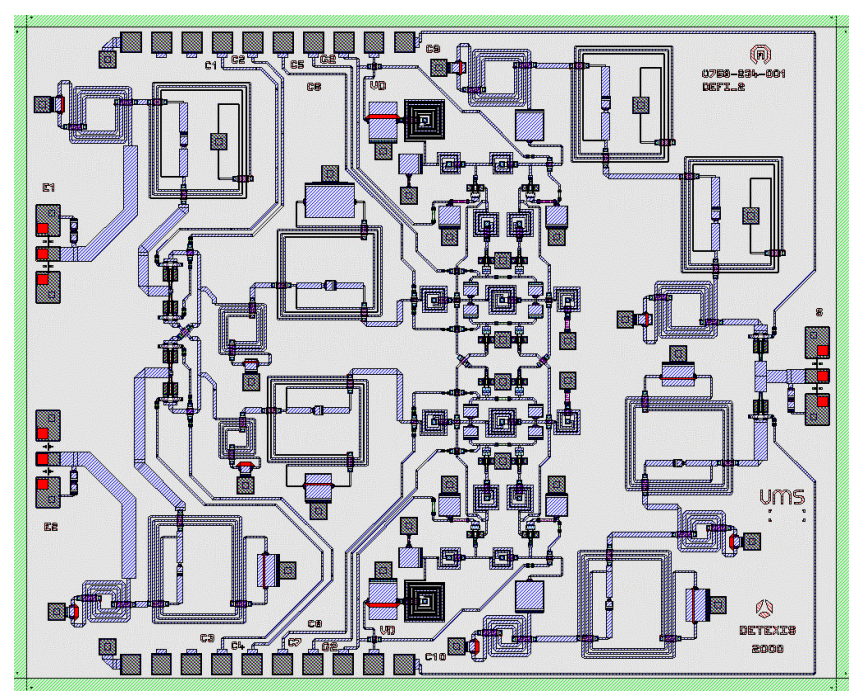

Figure 9: Layout of the second MMIC

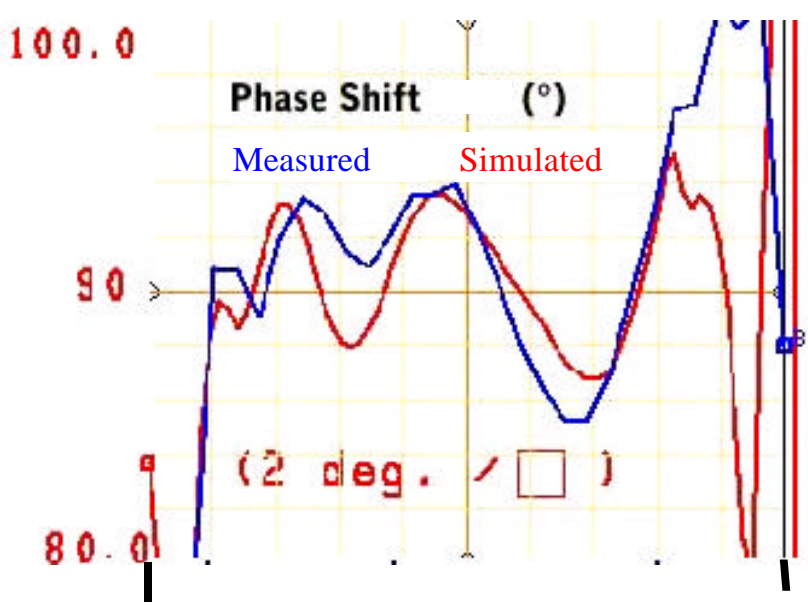

$500 \mathrm{MHz}$

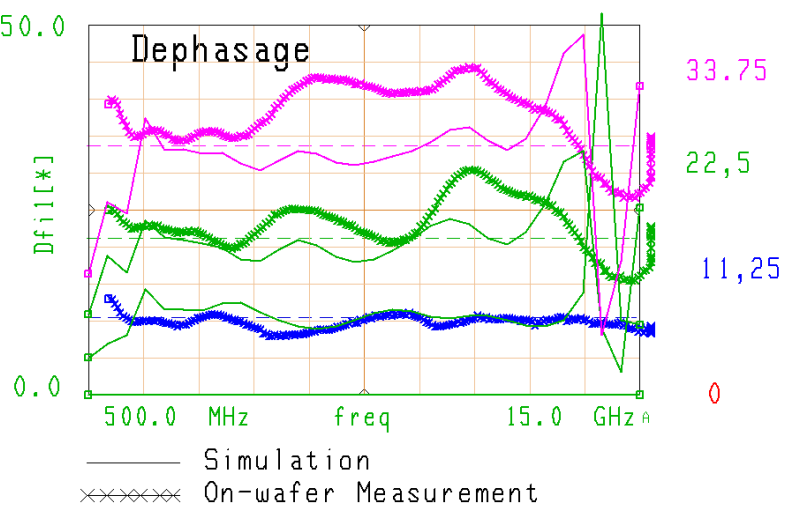

Figure 11 : Measured and simulated results for the first chip (lowest bits)

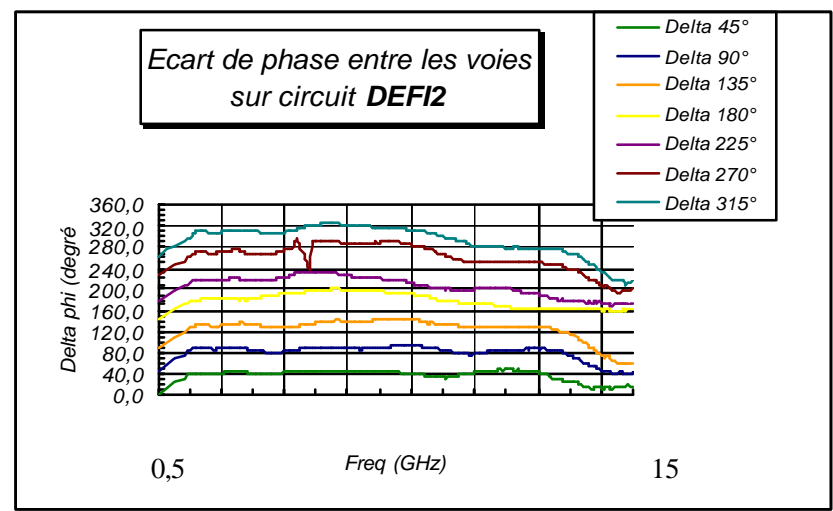

Figure $12:$ Measured results for the second chip (highest bits)

\section{CONCLUSION}

Innovative structures, including all-pass filters with interwoven spirals and active switches, have been developed to build an active broadband phase shifter with good results at the first foundry run. New refinements in simulation, using in particular full wave electromagnetic tools, will allow to design a second generation of circuits with improved performances.

Figure 10 : Example of measured versus simulated results for a single $90^{\circ}$ bit 